\title{
An Analytical study of a GaAs-Si n/p Heterojunction Solar Cell and Suggestion for A Structure for Improved Performance
}

\author{
Sayantan Biswas* \\ Research Scholar, Department of Physics, University of Kalyani, \\ Kalyani-741235, West Bengal, India. \\ Ashim Kumar Biswas \\ Assistant Professor, Department of Physics, \\ Nabadwip Vidyasagar College, Nabadwip-741302, West Bengal, India. \\ Research Scholar, Department of Physics, University of Kalyani, \\ Kalyani-741235, West Bengal, India.
}

Avigyan Chatterjee

Research Scholar, Department of Physics, University of Kalyani, Kalyani-741235, West Bengal, India.

\author{
Amitabha Sinha \\ Professor, Department of Physics, \\ University of Kalyani, Kalyani-741235, West Bengal, India.
}

\begin{abstract}
A theoretical investigation has been carried out on a $\mathrm{GaAs}-\mathrm{Si}$ $\mathrm{n} / \mathrm{p}$ heterojunction solar cell. Analytical expressions of the excess minority carrier concentration and photocurrent contribution from both the top and base regions of the cell have been obtained. The effects of different parameters such as front and back surface recombination velocities, wavelength of the incident light and corresponding absorption coefficient of the material on the excess minority carrier distribution and photocurrent density have been studied. It is observed from these theoretical studies that the back surface recombination has a significant effect on the photocurrent density and hence on the spectral response of these solar cells. Therefore, a structure of a heterojunction solar cell with a back surface field (BSF) has been suggested to give better performance, particularly with regard to the photocurrent of the cell.
\end{abstract}

Keywords: GaAs-Si heterojuction solar cell; minority carrier concentration; spectral response; surface recombination velocity.

\section{Introduction}

Heterojunctions are widely used in the designing of solar cells for the last few decades. Therefore lot of theoretical and experimental research work has been done on these solar cell structures. An analytical study on the conversion efficiency of heterojunction solar energy converters was reported by Shreedhar et al. [1]. They have calculated the efficiencies of various heterojunctions such as $\mathrm{Si}-\mathrm{GaAs}$ and $\mathrm{Si}-\mathrm{GaP}$. Several heterojunction solar cell efficiencies have been calculated by R. Sahai and A.G. Milnes [2], including the loss in efficiency due to reflection, incomplete collection and internal series resistance. Milnes and Feucht [3] discussed about different heterojunction pairs in detail. Effect of texturing and surface preparation on lifetime and cell performance in heterojunction solar cells have been studied in detail by M. Edwards et al. [4]. The surface recombination analysis in silicon heterojunction solar cell has been investigated theoretically by R. Barrio et al. [5]. The current losses at the front of silicon heterojunction solar cells have been discussed by Z.C. Holman et al. [6]. An analysis of the photovoltage of a heterojunction solar cell has been published by X. Chao [7]. Recently conversion efficiency over $20 \%$ on silicon heterojunction solar cells by IPA-free substrate texturization has been reported by J. Kegel et al. [8]. In this paper we have studied the excess minority carrier distribution in both the front and base regions of a GaAs-Si n/p heterojunction solar cell and also their contributions to the photocurrent densities of the cell, taking into account different solar cell parameters like surface recombination velocity, wavelength of the incident light and corresponding absorption coefficient of the material. Also, a new structure of the solar cell with a back surface field has been suggested to give better performance.

\section{Analysis}

In this paper $\mathrm{n} / \mathrm{p}$ GaAs-Si heterojunction solar cell structure has been considered. Schematic diagrams of $n / p$ GaAs-Si heterojunction solar cell and the coordinates assumed in the analysis have been shown in figure 1 . 


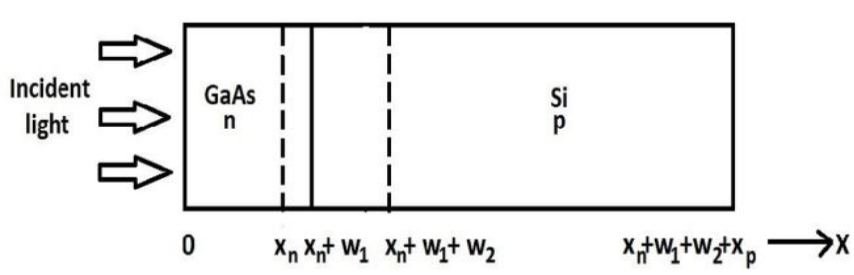

Figure 1: Schematic diagram of an $\mathrm{n} / \mathrm{p}$ heterojunction solar cell.

Analytical expressions for the minority carrier distribution and the photocurrent contribution from the top and the base regions of a n/p GaAs-Si heterojunction solar cell have been obtained. The mathematical approach given by H.J. Hovel [9] is followed for the calculation.

The generation rates of electron-hole pairs in the top and base regions of the cell are given by

$$
G_{p}=\alpha_{1}(\lambda) F(\lambda)[1-R(\lambda)] \exp \left[-\alpha_{1}(\lambda) x\right]
$$

and

$$
G_{\mathrm{n}}=\alpha_{2}(\lambda) F(\lambda)[1-R(\lambda)] \exp \left[-\alpha_{2}(\lambda) x\right]
$$

where $\alpha_{1}$ and $\alpha_{2}$ are the absorption coefficients of material-1 and material-2 of the cell respectively.

Combining these equations with the continuity and current density equations, the expression for the hole concentration in the front layer may be written as

$$
\begin{gathered}
\Delta p_{n p}=\left[\frac{\alpha_{1} F(1-R) \tau_{p}}{\left(\alpha_{1}^{2} L_{p}^{2}-1\right)}\right] \times \\
{\left[\frac{\left(\frac{S_{p} L_{p}}{D_{p}}+a_{1} L_{p}\right) \sinh \left(\frac{x_{p}-x}{L_{p}}\right)+\exp \left(-a_{1} x_{n}\right)\left(\frac{S_{p} L_{p}}{D_{p}} \sinh \left(\frac{x}{L_{p}}\right)+\cosh \left(\frac{x}{L_{p}}\right)\right\}}{\frac{S_{p} L_{p}}{D_{p}} \sinh \left(\frac{x_{p}}{L_{p}}\right)+\cosh \left(\frac{x_{p}}{L_{p}}\right)}-\exp \left(-a_{1} x\right)\right]}
\end{gathered}
$$

Where the following boundary conditions have been used [9]

$$
\begin{aligned}
& \text { at } \mathrm{x}=0 D_{\mathrm{p}} \frac{\left.d \& \Delta p_{n}\right)}{d x}=S_{p} \Delta p_{n} \\
& \text { at } \mathrm{x}=\mathrm{x}_{\mathrm{n}} \Delta p_{\mathrm{n}}=0
\end{aligned}
$$

The photocurrent density of the front region is [9]

$$
\begin{gathered}
I_{p}=\left[\frac{q F(1-R) \alpha_{1} L_{p}}{\left(\alpha_{1}^{2} L_{p}^{2}-1\right)}\right] \times \\
{\left[\frac{\left(\frac{S_{p} L_{p}}{D_{p}}+a_{1} L_{p}\right)-\exp \left(-a_{1} x_{n}\right)\left(\frac{S_{p} L_{p}}{D_{p}} \cosh \left(\frac{x_{p}}{L_{p}}\right)+\sinh \left(\frac{x_{p}}{L_{p}}\right)\right)}{\frac{S_{p} L_{p}}{D_{p}} \sinh \left(\frac{x_{p}}{L_{p}}\right)+\cosh \left(\frac{x_{p}}{L_{p}}\right)}-a_{1} L_{p} \exp \left(-a_{1} x_{n}\right)\right]}
\end{gathered}
$$

Therefore the spectral response of the top region is given by

$$
[S R]_{\text {top }}=\left|\frac{J_{p}}{q F(1-R)}\right|
$$

Similarly, the electron concentration in the base region may be obtained by combining the continuity equation and the current density equation and using the following boundary conditions [9]

$$
\begin{aligned}
& \text { at } x=x_{n}+w_{1}+w_{2} \Delta n_{p}=0 \\
& \text { at } x=x_{n}+w_{1}+w_{2}+x_{p} D_{n} \frac{d \Delta n_{p}}{d x}=\Delta n_{p}
\end{aligned}
$$

The electron concentration in the base layer may be thus written as

$$
\begin{aligned}
& \Delta n_{p}= {\left[\frac{\alpha_{2} F(1-R) \tau_{n} \exp \left(-\alpha_{2} w_{2}\right) \exp \left(-\alpha_{1}\left(x_{n}+w_{1}\right)\right]}{\left(\alpha_{2}^{2} L_{n}^{2}-1\right)}\right] \times } \\
& {\left[\cosh \left(\frac{x-x_{n}-w_{1}-w_{2}}{L_{n}}\right)-\exp \left\{-a_{2}\left(x-x_{n}-w_{1}-w_{2}\right)\right]\right.} \\
&\left.\quad-\frac{\left.\left[\frac{S_{n} L_{n}}{D_{n}}\left\{\cosh \left(\frac{x_{p}}{L_{n}}\right)-\exp \left(-a_{2} x_{p}\right)\right\}+\sinh \left(\frac{x_{p}}{L_{n}}\right)+a_{2} \exp \left(-a_{2} x_{p}\right)\right] \sinh \left(\frac{x-x_{n}-w_{1}-w_{p}}{L_{n}}\right)\right]}{\frac{S_{n} L_{n}}{D_{n}} \sinh \left(\frac{x_{p}}{L_{n}}\right)+\cosh \left(\frac{x_{p}}{L_{n}}\right)}\right] \text { (10) }
\end{aligned}
$$

The corresponding photocurrent density from the base region is given by [9]

$$
\begin{aligned}
& I_{n}=\left[\frac{q \alpha_{2} F(1-R) L_{n} \exp \left(-\alpha_{2} w_{2}\right) \exp \left(-\alpha_{1}\left(x_{n}+w_{1}\right)\right)}{\left(\alpha_{2}^{2} L_{n}^{2}-1\right)}\right] \times \\
& {\left[a_{2} L_{n}-\frac{\frac{S_{n} L_{n}}{D_{n}}\left\{\cosh \left(\frac{x_{p}}{L_{n}}\right)-\exp \left(-a_{2} x_{p}\right)\right\}+\sinh \left(\frac{x_{p}}{L_{n n}}\right)+a_{2} L_{n} \exp \left(-\alpha_{2} x_{p}\right)}{\frac{S_{n} L_{n}}{D_{n}} \sinh \left(\frac{x_{p}}{L_{n}}\right)+\cosh \left(\frac{x_{p}}{L_{n}}\right)}\right] \text { (11) }}
\end{aligned}
$$

Hence the spectral response of the base region is given by

$$
[S R]_{\text {bnge }}=\left|\frac{J_{n}}{q F(1-R)}\right|
$$

The depletion widths $\mathrm{w}_{1}$ and $\mathrm{w}_{2}$ are determined by the following equations [9]

$$
\begin{aligned}
& w_{1}=\left[\left(\frac{2 \epsilon_{1} v_{d}}{q N_{1}}\right)\left(\frac{\epsilon_{2} N_{2}}{\epsilon_{1} N_{1}+\epsilon_{2} N_{2}}\right)\right]^{\frac{1}{2}} \\
& w_{2}=\left[\left(\frac{2 \epsilon_{2} v_{d}}{q N_{2}}\right)\left(\frac{\epsilon_{1} N_{1}}{\epsilon_{1} N_{1}+\epsilon_{2} N_{2}}\right)\right]^{\frac{1}{2}}
\end{aligned}
$$

where $\mathrm{N}_{1}$ and $\mathrm{N}_{2}$ are the densities of acceptor and donor atoms respectively, $\epsilon_{1}$ and $\epsilon_{2}$ are the relative permittivities of material- 1 and material- 2 respectively and $v_{d}$ is the barrier height.

\section{Results and Discussions}

Based on the analytical expressions obtained in the preceding section, calculations were performed and the results are shown in the figures. The concentration of the donor atoms taken in the calculations for the top region is $1.7 \times 10^{17} \mathrm{~cm}^{-3}$ and the concentration of the acceptor atoms in the base region is taken as $10^{15} \mathrm{~cm}^{-3}$. The diffusion length and diffusion constant for the holes in the top region are taken as $3 \mu \mathrm{m}$ and $10 \mathrm{~cm}^{2} \mathrm{~s}^{-1}$ respectively. The same for the electrons in the base region are taken as $225.7 \mu \mathrm{m}$ and $34.2 \mathrm{~cm}^{2} \mathrm{~s}^{-1}$ respectively following the relationships given by J.G. Fossum [10]. The width of the top region is taken as $0.6 \mu \mathrm{m}$ and that of the base region is $100 \mu \mathrm{m}$.

Using equation (3), the excess minority carrier hole concentration of the top region of a n/p GaAs-Si heterojunction solar cell has been calculated and the results have been plotted in Fig.2 for various values of front surface recombination velocity $S_{p}$. It is observed that the minority carrier concentration is much higher near the surface, for low 
front surface recombination velocity $\mathrm{S}_{\mathrm{p}}$ and lower for higher values of $S_{p}$. This is due to the reason that as a result of recombination at the front surface, many minority carriers are lost there.

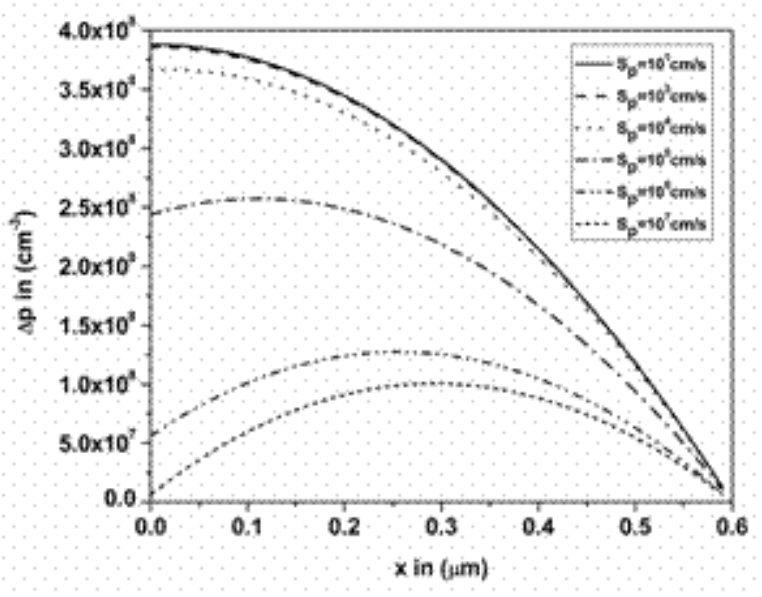

Figure 2: Variation of excess minority carrier concentration with position corresponding to different values of front surface recombination velocity $S_{p}$ of the top region.

Fig. 3 represents the spectral response contribution of the top region for different values of front surface recombination velocity $S_{p}$. It is observed from the figure that the photocurrent contribution is much higher for smaller values of $S_{\mathrm{p}}$. This is interpreted from the fact that for smaller values of $\mathrm{S}_{\mathrm{p}}$ the recombination is low at the front surface and this result in increased value of photocurrent contribution from this region. Also it is observed that the spectral response attains maximum value for the wavelength $\lambda$ about $5000 \AA$.

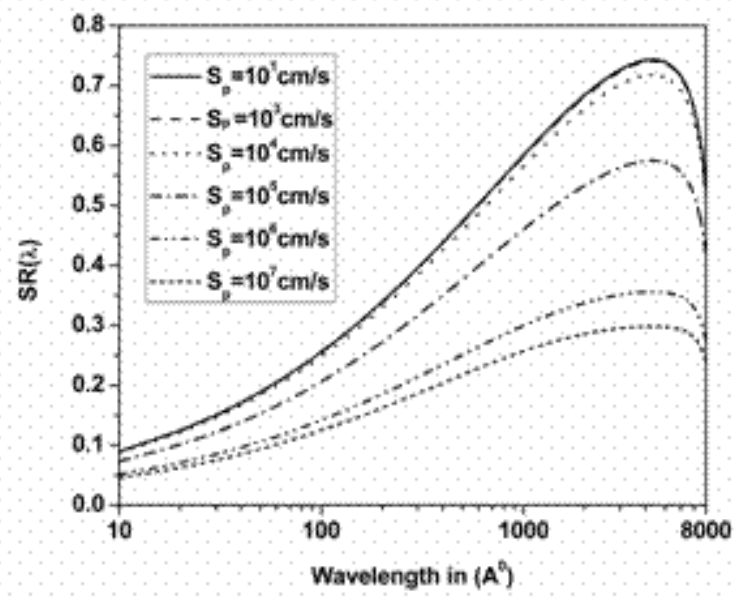

Figure 3: Variation of spectral response with wavelength of the top region corresponding to different values of front surface recombination velocity $S_{p}$.

Fig.4 shows the variation of excess carrier concentration in the p-type base region of the solar cell as a function of position for different values of the back surface recombination velocity $S_{n}$. It is observed that the minority carrier concentration is much higher near the rear surface of the cell for smaller values of rear surface recombination velocity $S_{n}$. This is interpreted from the fact that low recombination at the rear leads to large accumulation of the carriers there.

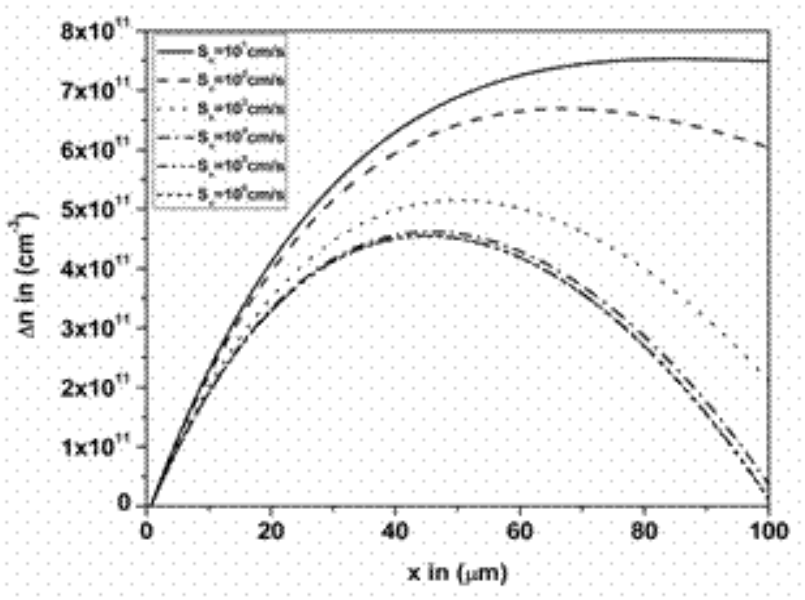

Figure 4: Variation of excess minority carrier concentration with position corresponding to different values of rear surface recombination velocity $S_{n}$ of the base region.

Fig. 5 shows the variation of the spectral response of the base region of the cell as a function of wavelength $\lambda$ for different values of rear surface recombination velocity $S_{n}$. From the figure it is observed that the spectral response of the base region is significantly dependent on the back surface recombination velocity $S_{n}$.

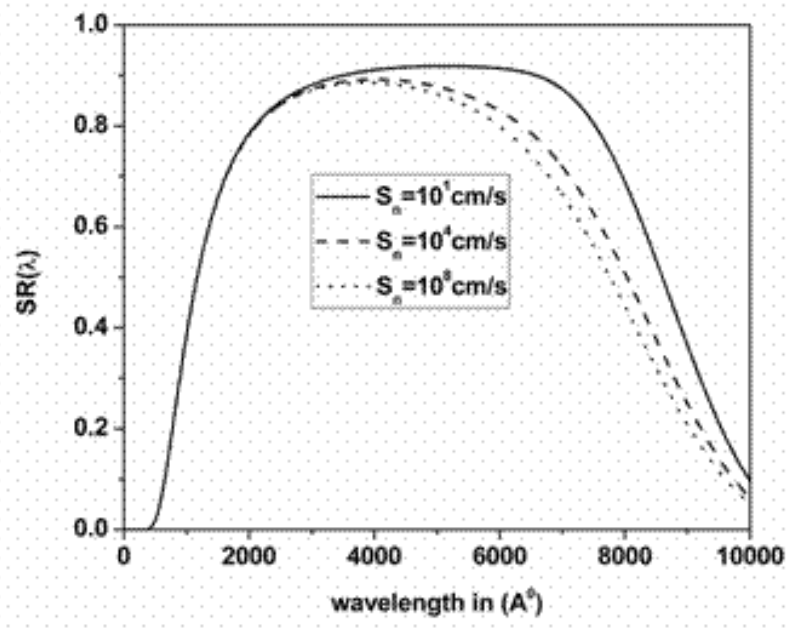

Figure 5: Variation of spectral response with wavelength of the base region corresponding to different values of back surface recombination velocity $S_{n}$.

\section{Suggestion for a new heterojunction solar cell structure with a back surface field}

It is observed from Fig.5 that the spectral response of the base 
layer of the solar cell improves significantly as the back surface recombination decreases. Keeping in view this observation, we suggest a new heterojunction solar cell structure with a back surface field, as shown in Fig.6.

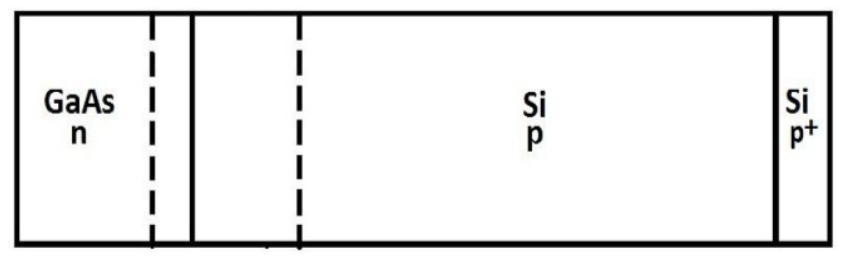

Figure 6: Schematic diagram of the suggested GaAs-Si n on $\mathrm{p}$ heterojunction solar cell.

It was observed in the case of conventional $n$ on $p$ silicon solar cells that incorporating a $\mathrm{p}^{+}$layer at its back gave rise to $\mathrm{a} \mathrm{n}^{+} \mathrm{p} \mathrm{p}^{+}$silicon solar cell which provided a back surface field (BSF) and increased the efficiency of the cell significantly $[11,12]$. From the same analogy, we expect that the new structure suggested by us in Fig.6 would give much improved performance as compared to conventional heterojunction solar cell as shown in Fig.1.

It may be mentioned here that similar structure of a schottky barrier solar cell has been suggested recently to yield improved performance [13].

\section{Conclusion}

The excess minority carrier distribution in the top and base regions of a GaAs-Si n/p heterojunction solar cell has been studied and their contributions to the photocurrent of the device has been investigated, taking into account the front and back surface recombination velocities. It is observed from the results that for smaller values of the recombination velocities the photocurrent and the spectral response increase. This implies the important role of back surface field (BSF) in these structures. Hence a new structure of a heterojunction solar cell with a BSF has been suggested here to get improved performance.

\section{Acknowledgements}

We are grateful to the Department of Science and Technology, Govt. of India, for financial support under the DST-PURSE Programme, granted to the University of Kalyani. We thank the authorities of Indian Association for the Cultivation of Science, Kolkata, India, for allowing us to consult their library.

\section{References}

[1] Shreedhar, A.K., Sharma, B.L., and Purohit, R.K., 1969, "Efficiency calculations of heterojunction solar energy converters," IEEE Trans. Electron Dev., 16, pp. 309-312.

[2] Sahai, R., and Milnes, A.G., 1970, "Heterojunction Solar Cell Calculations," Solid State Electronics, 13, pp. 1289-1299.

[3] Milnes, A.G., and Feucht, D.L., 1972, "Heterojunction and Metal-Semiconductor Junctions," Academic Press, New-York.

[4] Edwards, M., Bowden, S., Das, U., and Burrows, M., 2008, "Effect of texturing and surface preparation on lifetime and cell performance in heterojunction silicon solar cells," Solar Energy Materials and Solar Cells, 92, pp. 1373-1377.

[5] Barrio, R., Gandia, J.J., Carabe, J., Gonzalez, N., Torres, I., Munoz, D., and Voz, C., 2010, "Surface recombination analysis in silicon-heterojunction solar cells," Solar Energy Materials and Solar Cells, 94, pp. 282-286.

[6] Holman, Z.C., Descoeudres, A., Barraud, L., Farnandez, F.Z., Seif, J.P., Wolf, S.De, and Ballif, C., 2012, "Current Losses at the Front of Silicon Heterojunction Solar Cells," IEEE Journal of Photovoltaics, 2, pp. 7-15.

[7] Chao, X., He, Y.R., and Wei, G.K., 2011, "Photovoltage analysis of a heterojunction solar cell," Chin Phys. B, 20, pp. 057302.

[8] Kegel, J., Angermann, H., Sturzebecher, U., Conrad, E., Mews, M., Korte, L., and Stegemann, B., 2014, "Over 20\% conversion efficiency on silicon heterojunction solar cells by IPA-free substrate texturization," Applied Surface Science, 301, pp. 5662.

[9] Hovel, H.J., 1975, "Semiconductors and Semimetals: Solar Cells," 11, Academic Press, New York, pp. 127-139.

[10] Fossum, J.G., 1976, "Computer-Aided Numerical Analysis of Silicon Solar Cells," Solid State Electronics, 19, pp. 269-277.

[11] Mandelkorn, J., and Lamneck Jr., J.H., 1973, “A new electric field effect in silicon solar cells," J. Appl. Phys., 44, pp. 4785-4787.

[12] Sinha, A., and Chattopadhyaya, S.K., 1978, "Effect of heavy doping on the properties of high-low junction," IEEE Trans. Electron Dev., 25, pp. 14121414.

[13] Chatterjee, A., Biswas, A. K., and Sinha, A., 2015, "Dependence of the photocurrent of a schottkybarrier solar cell on the back surface recombination velocity and suggestion for a structure with improved performance," Journal of Solar Energy, 2015, Article ID 252916 . 